\title{
Les mariages des ténèbres
}

Images votives d'unions post-mortem au Japon

\section{Agnès Giard}

\section{OpenEdition}

\section{Journals}

Édition électronique

URL : https://journals.openedition.org/tc/10184

DOI : $10.4000 /$ tc. 10184

ISSN : 1952-420X

Éditeur

Éditions de l'EHESS

\section{Édition imprimée}

Date de publication : 30 octobre 2018

Pagination : 168-173

ISBN : 2-7132-2751-6

ISSN : 0248-6016

Référence électronique

Agnès Giard, «Les mariages des ténèbres », Techniques \& Culture [En ligne], 70 | 2018, mis en ligne le 06 décembre 2018, consulté le 29 septembre 2022. URL : http://journals.openedition.org/tc/10184 ; DOI : https://doi.org/10.4000/tc.10184 


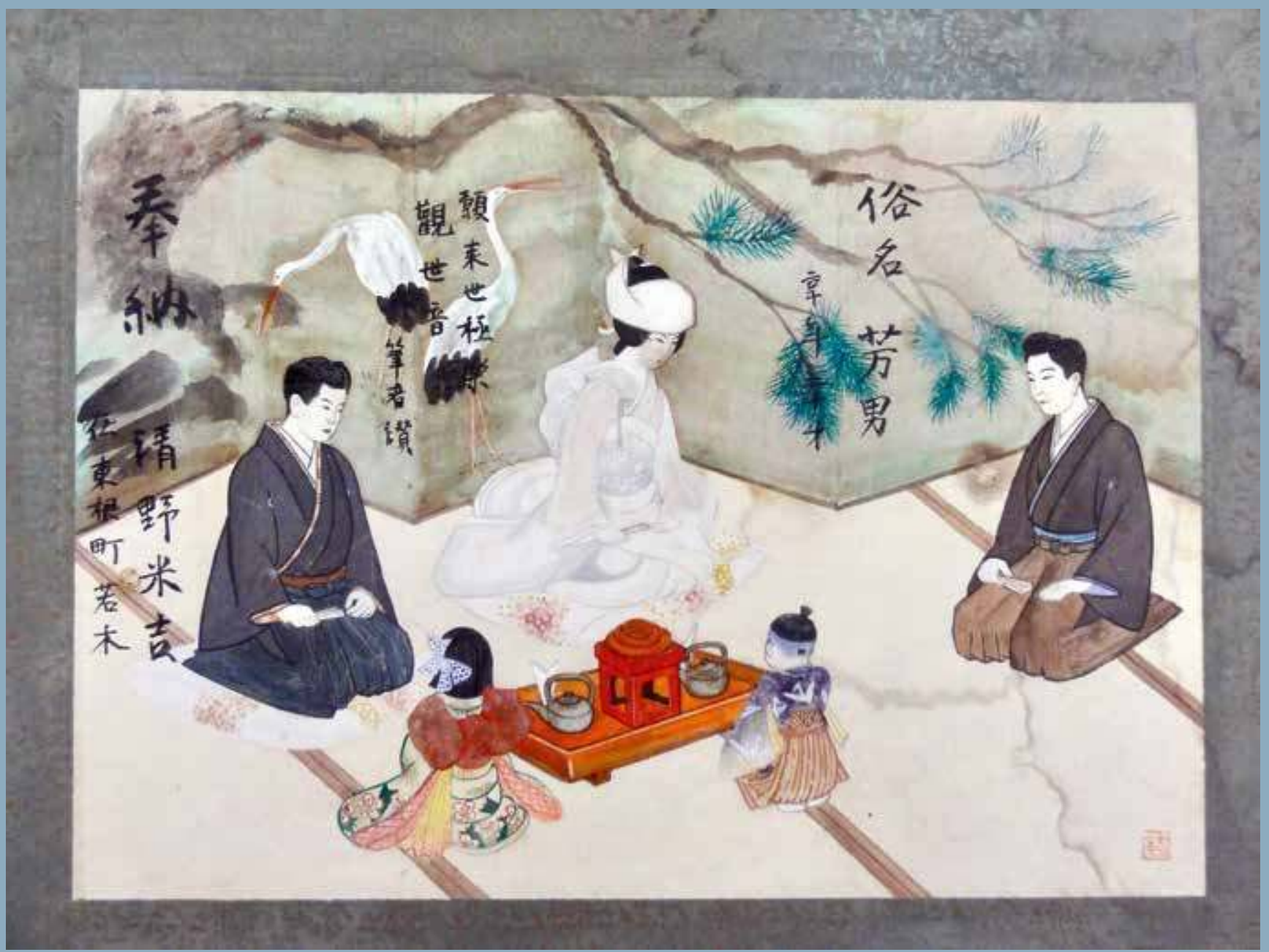




\section{Les mariages des ténèbres} Images votives d'unions post-mortem au Japon

Il existe au nord du Japon une coutume peu connue appelée «mariage des ténèbres». Cette coutume veut que si une personne décède sans s'être mariée, sa famille lui organise un mariage post-mortem afin de la rendre heureuse. Il faut que les esprits des morts trouvent la paix dans l'autre monde. Si ces morts restaient seuls, frustrés d'amour et privés d'une descendance qui leur consacre des rites, ils seraient susceptibles de devenir des âmes en peine. Incapables de se détacher de notre monde, tourmentés par l'angoisse, ils pourraient se transformer en êtres dangereux, c'està-dire entraîner les vivants dans la spirale de leur douleur. Ces morts-là sont doués du pouvoir d'affecter les vivants. Qu'il s'agisse d'enfants morts trop jeunes ou de célibataires âgés - quelles que soient les raisons pour lesquelles ils et elles n'ont jamais fondé un foyer (pauvreté, handicap, décès précoce ou autre) -, ces personnes qui appartiennent à la catégorie des «bouddhas sans lien» (muen-botoke) sont considérées comme hautement toxiques et c'est pourquoi toutes sortes de rituels leur sont dédiés afin qu'elles puissent, dans l'au-delà, trouver une forme de délivrance. La coutume du mariage des ténèbres fait partie des multiples moyens d'aider ces morts à "partir». Elle s'appuie sur l'offrande d'ex-voto singuliers - les mukasari ema, « images votives de mariage »qui représentent la personne morte, célébrant son union avec une personne imaginaire.

À la différence des ex-voto habituels qui sont offerts pour l'accomplissement d'un vœu (trouver de l'argent, par exemple) ou en remerciement d'une grâce obtenue (survivre à une catastrophe...), les mukasari ema servent à exprimer des souhaits pour le bénéfice non pas des vivants mais des morts. Il peut paraître incongru de les traiter dans ce dossier car les ex-voto, en général, sont donnés en échange d'une vie sauvée, ou d'une vie protégée, mais d'une vie. Pas d'une mort. Jusqu'à présent, les chercheurs se sont surtout intéressés aux ex-voto comme à des outils de transaction entre les vivants et les divinités, transaction visant le bonheur ici-bas, sans prendre en compte le cas particulier de ces morts qui, quoique disparus, continuent de hanter les mémoires et avec lesquels il faut bien vivre. Même invisibles, ces morts font partie de la communauté et c'est 


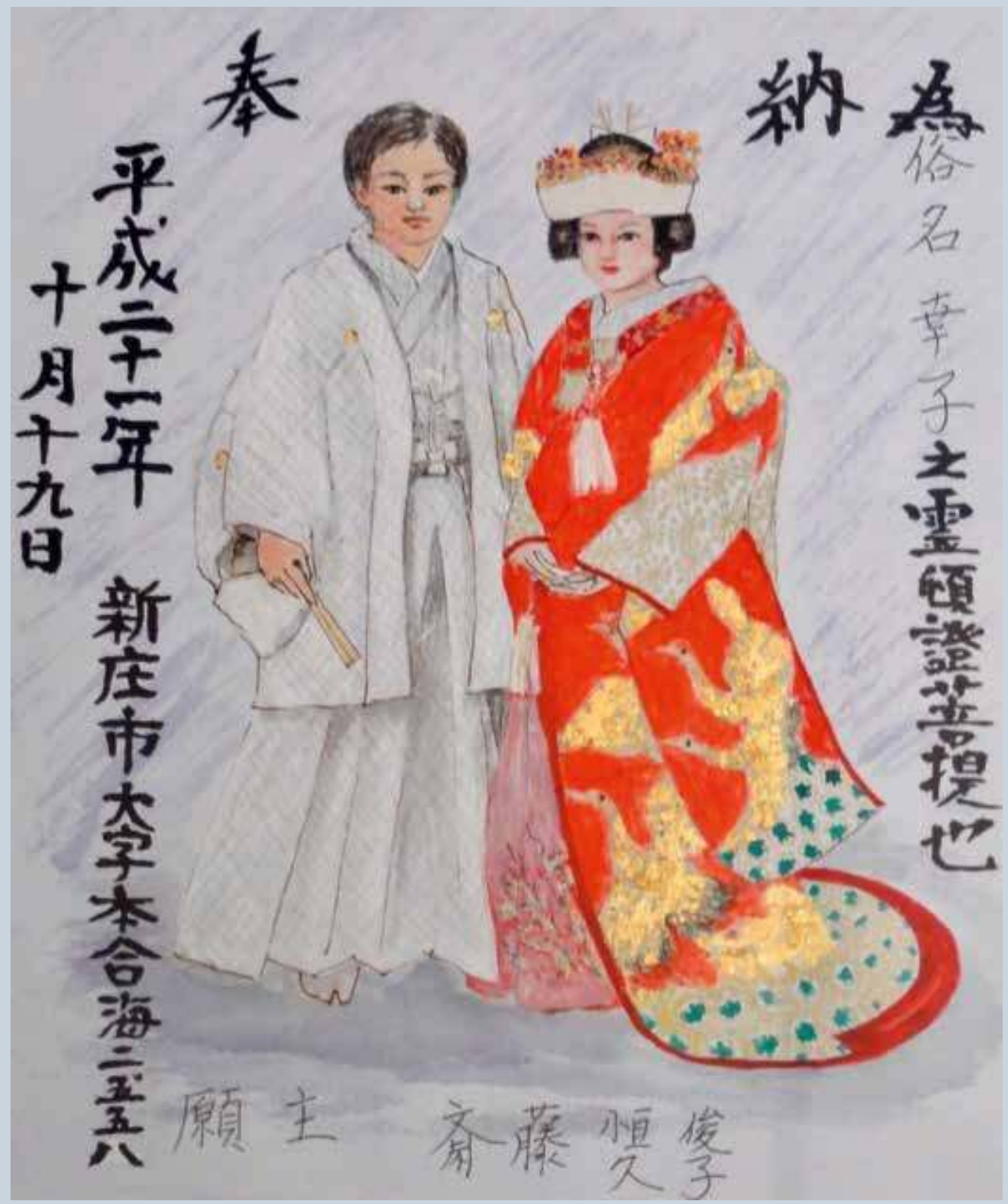

Mukasari ema amateur daté de 2009 


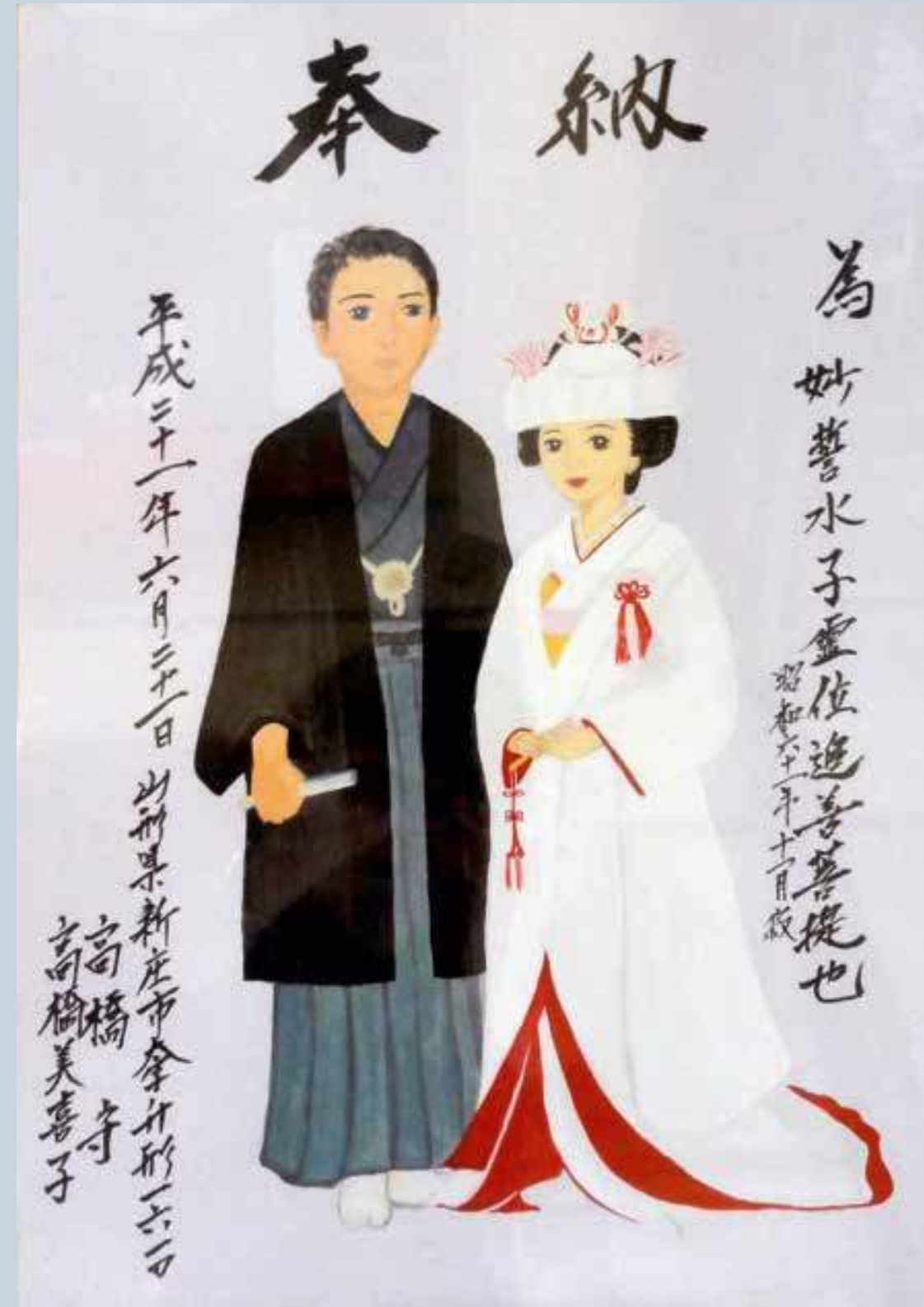


3. Mukasari ema daté de 1934

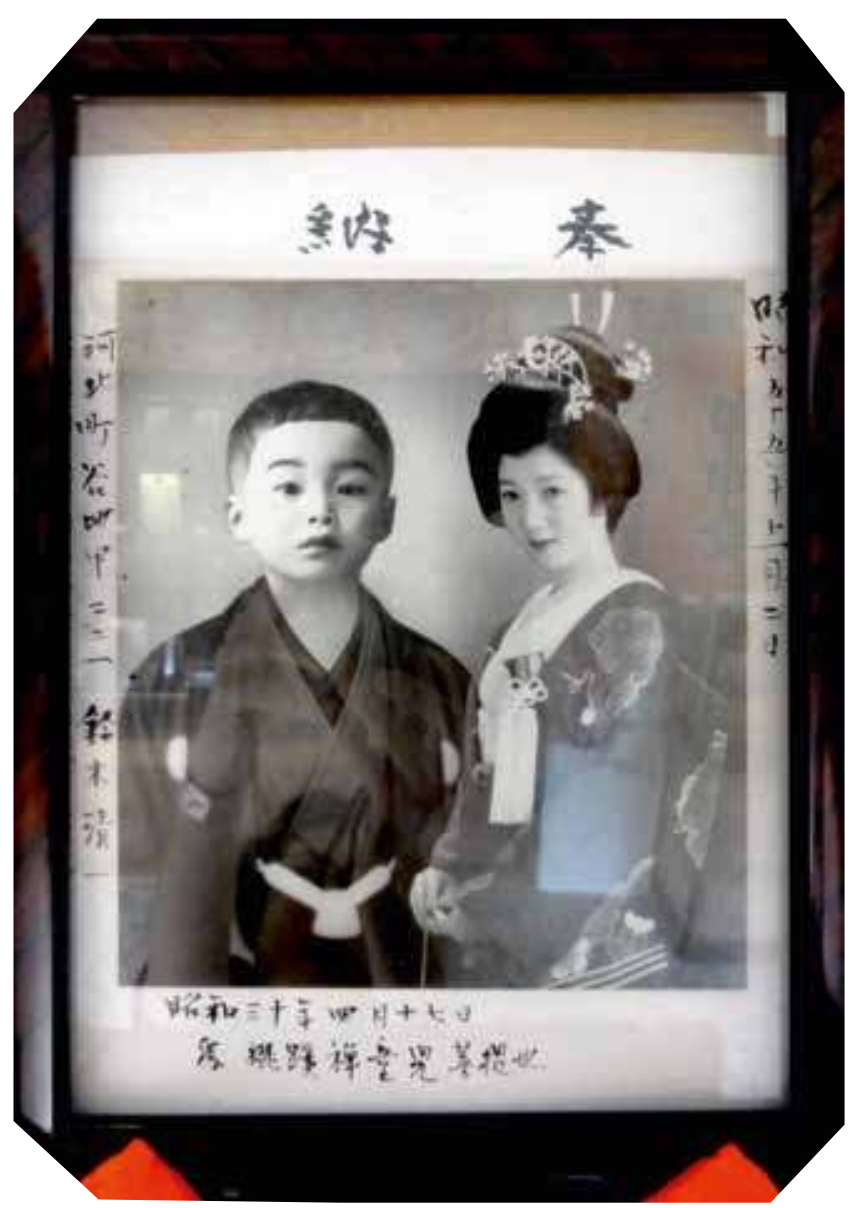

pourquoi les offrandes votives qui sont faites pour leur bénéfice servent tout autant les intérêts des vivants. Les mukasari ema apportent un éclairage utile sur la diversité des stratégies dont les ex-voto se font les instruments: dans ce cas très particulier, la stratégie consiste non seulement à offrir un mariage à l'esprit du mort, mais à offrir l'image votive de ce mariage à un temple.

Au Japon, seuls huit temples bouddhiques - situés dans la localité de Murayama - accueillent de telles images. Le plus connu d'entre eux, le Wakamatsu, possède plus de 1000 mukasari ema. Lorsqu'un mariage des ténèbres est célébré, cela se passe ainsi: la famille en deuil, parfois juste un parent, vient avec un mukasari ema représentant la personne disparue. Qu'il soit fait maison, colorié maladroitement, ou réalisé sur commande par un artiste spécialisé dans ce type de production, le mukasari ema possède la même efficacité. Peu importe le résultat esthétique, pourvu que l'image soit «chargée de pensées» (omoi o takushite), pourvu qu'elle ait été faite en pensant très fort à l'absent•e. Les règles qui président à l'élaboration du mukasari ema se résument de fait à une seule: il ne faut jamais mettre en scène une personne réelle, vivante, aux côtés de la personne disparue. Les frontières entre les mondes doivent rester étanches. Pour donner à l'image votive la qualité d'une féerie, le mort (il peut s'agir du fils, de la fille, du frère, de la søur, d'une tante ou d'un oncle) est donc représenté à l'aide d'un dessin, d'une peinture ou d'un collage bariolé reproduisant les fastes de l'union matrimoniale. Costume pimpant aux couleurs de la fête, image idéalisée d'un jeune couple... Aux côtés du mort (de la morte) son épouse ou son époux de rêve sourit en regardant le spectateur, comme sur les albums photo de mariage.

En 2017, un journaliste publie sur la version japonaise de Yahoo News un reportage concernant les mukasari ema. Il rapporte le témoignage suivant: «Dans la ville d'Iwata, une femme dont le frère - âgé de 18 ans - fut brutalement emporté par la mort lui fit faire, trois ans plus tard, un mukasari ema en guise d'offrande funéraire. » Pourquoi attendit-elle trois ans avant de faire le mukasari ema? L'histoire, ici, devient étrange: cette femme anonyme raconte qu'au moment des funérailles, elle avait la sensation perturbante que quelque chose n'allait pas. «Le service s'était achevé, mais elle sentait toujours la présence de son frère à ses côtés. » Puis, une nuit, elle fit un rêve... Le mort lui apparut: «J'aimerais aller dans un endroit plus 
lumineux. Ici, il fait froid», dit-il d'une voix plaintive, avant d'ajouter: « Le service commémoratif n'a pas été célébré correctement. » Le rituel avait pourtant été exécuté dans les règles de l'art. Que faire? «La souffrance de son frère mort lui pesait sur le cœur, mais elle ne savait pas quel rituel serait capable de l'alléger. » Cela dura ainsi plusieurs années, jusqu'à ce que la femme apprenne à la télévision l'existence des mukasari ema. Elle téléphona au temple Wakamatsu. On lui indiqua le contact d'une artiste spécialisée, Takahashi Chikako. Celle-ci dessina son frère souriant, en compagnie d'une «adorable épouse». Une fois achevée, l'image fut encadrée sous verre puis accrochée dans la salle des prières du temple Wakamatsu au cours d'une cérémonie funéraire. «La femme prit une photo de l'ex-voto dédié au mort puis, regardant la photo, incapable de se contenir, elle pleura. Le soir, alors qu'elle s'était endormie, son frère lui apparut tel qu'il était avant sa mort, il lui sourit, puis disparut. "Je suis venu te dire Merci". » Son frère n'est plus jamais revenu la voir.

De façon significative, la coutume des mukasari ema connaît un regain de popularité car un nombre croissant de Japonais•es font appel à Takahashi Chikako pour entrer en contact avec l'âme d'une personne disparue et régler leurs propres problèmes affectifs par le biais de ces images votives qui symbolisent le nouveau départ. Les prêtres bouddhistes encouragent volontiers ces pratiques qui -à mi-chemin entre la nécromancie et la thérapie transgénérationnelle-contribuent à la popularité de leur temple et attirent un public nouveau en quête de rituels religieux alternatifs.

\section{Remerciements}

De l'auteure à Élina Meyer, pour son travail d'accompagnement lors de cette recherche.

\section{En ligne}

Retrouver l'article complet sur journals.openedition.org/tc : TechniquesECulture 70 « Matérialiser les désirs».

\section{Iconographie}

Image d'ouverture. Mukasari ema sans date. (C) Agnès Giard, Jakushô, juillet 2012.

Crédits pour l'ensemble des illustrations. (C) Agnès Giard, Jakushô, juillet 2012.

\section{I'auteure}

Agnès Giard est anthropologue, chercheur rattaché au laboratoire Sophiapol (EA 3932) université de Paris Nanterre. Ses recherches en anthropologie portent sur l'industrie des simulacres affectifs (low-tech/high-tech) dans le contexte du dépeuplement du Japon. Elles mettent en regard la consommation des produits de consommation émotionnels et le stigmate frappant les personnes qui vivent seules, ainsi que la perspective d'une mort sans descendance.

\section{Pour citer l'article}

Giard, A. 2018 «Les mariages des ténèbres. Images votives d'unions post-mortem au Japon », Techniques\&Culture 70 «Matérialiser les désirs. Techniques votives», p. 168-173. 